\title{
Magnetic fields in dwarfs versus early-type galaxies
}

\author{
Krzysztof T. Chyży
}

Astronomical Observatory, Jagiellonian University, ul. Orla 171, 30-244 Kraków, Poland; email: chris@oa.uj.edu.pl

\begin{abstract}
According to a recent systematic study of dwarf irregular galaxies the production of their magnetic fields appears to be regulated mainly by the surface density of the galactic star-formation rate. Magnetic fields in nearby dwarfs are typically weak, with the mean value of the total field strength three times smaller than in the normal spirals. Dwarfs with stronger fields reveal vivid star-forming activity, have clear signs of current or recent gravitational interactions, are more massive and evolved systems. Recently discovered strong regular fields in an early-type ringed galaxy NGC 4736 also indicates that even without spiral density waves an effective generation of strong magnetic fields is possible in any type of galaxy if only starburst characteristics are reached.
\end{abstract}

Keywords. galaxies: irregular, galaxies: dwarf, galaxies: starburst, galaxies: magnetic fields

\section{Generation of magnetic fields in dwarfs}

It is well known that dwarf galaxies are the most numerous species in the Universe. But they are also tiny and weak objects, rather difficult to observe, especially in the radio domain. Therefore, it is not well known whether strong magnetic fields could be easily generated in such low-mass objects. Surprisingly, in an optically bright, dwarf irregular galaxy NGC 4449, a very strong radio polarized emission was detected 2000. With the strength of the total magnetic field of about $12 \mu \mathrm{G}$ and of the regular component about $8 \mu \mathrm{G}$, it is very similar to typical massive and grand-design spiral galaxies (Beck 2005). Are such strong magnetic fields a rule or an exception among the dwarf galaxies?

The recent systematic study of radio emission and polarization in nearby dwarf galaxies indicates that their magnetic fields are predominately weak (Chyży et al. 2009). The mean value of the total magnetic field strength is $4 \mu \mathrm{G}$, three times smaller than in the normal spirals. It is found that magnetic field strength depends primarily on the surface density of the galactic star formation rate. The strongest magnetic fields in the nearby dwarfs have been detected only in highly evolved, most massive dwarfs, showing also clear effects of past interactions. Such interactions could probably trigger their star formation bursts. In such conditions of enhanced star-forming activity strong magnetic fields may effectively be produced through a small-scale dynamo process.

It seems that the high-redshift dwarfs could have a considerably higher star formation rate than the local analogs and thus could also have stronger magnetic fields or even totally regular ones (Arshakian et al. 2009). However, in such a case distant dwarfs would be completely different from the local ones, great majority of which show only weak magnetic fields.

\section{Dwarfs versus early-type galaxies}

The dependence of the magnetic field strength upon the star formation rate is observed not only for low-mass systems. It is also well visible in case of early-type galaxies. NGC 4736, for example, reveals a ringed morphology caused by the inner Lindblad resonance due to the galactic oval. The resonance causes the accumulation of gas and triggers star formation in the ring. It is the only region within the galaxy where young stars are produced. Inside and around the ring magnetic fields are exceptionally strong reaching $30 \mu \mathrm{G}$ (Chyży \& Buta 2008). They even ignore 


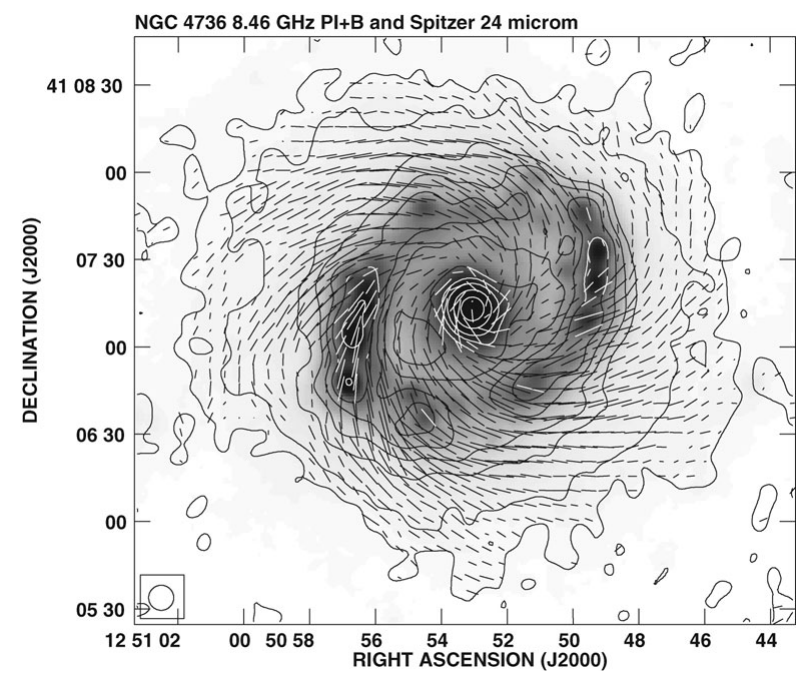

Figure 1. The radio intensity contour map of NGC 4736 at $8.46 \mathrm{GHz}$ and 8."6x8." 6 resolution with observed magnetic field vectors of the polarized intensity superimposed upon the infrared $24 \mu \mathrm{m}$ image (from Spitzer survey of SINGS galaxies: Kennicutt et al. 2003). The contours are at $0.02,0.06,0.19,0.30,0.48,0.77,1.54 \mathrm{mJy} /$ beam area.

the ring morphology and cross it at a large pitch angle forming a very coherent spiral pattern (Fig. 1).

If we compare NGC 4736 with the Sombrero galaxy, which is another early-type object, clear differences are to be seen. In the Sombrero galaxy, which produces young stars ten times slower than NGC 4736, only some weak patches of magnetized medium with strength of up to $6 \mu \mathrm{G}$ were detected (Krause et al. 2006). Hence, even without spiral density waves an effective generation of strong magnetic fields is still possible in an early-type galaxy if only starburst characteristics are attained. This resembles the starbursting dwarfs, which also show strong fields, but here the starbursting ring is triggered not by gravitational interactions like in dwarfs but by the Lindblad resonance.

Acknowledgements This work was supported by the Polish Ministry of Science and Higher Education through grants: 92/N-ASTROSIM /2008/0 and 3033/B/H03/2008/35.

\section{References}

Arshakian, T. G., Beck, R., Krause, M., \& Sokoloff, D. 2009, A\&A 494, 21

Beck, R. 2005, In: Cosmic Magnetic Fields, eds. R. Wielebinski \& R. Beck, Springer, p. 41

Chyży, K. T., Beck, R., Kohle, S., Klein, U., \& Urbanik, M. 2000, A\&A 355, 128

Chyży, K. T., \& Buta, R. J. 2008, ApJ 677, L17

Chyży, K. T., Weżgowiec, M., Beck, R., \& Bomans, D. J. 2009, A\&A, to be submitted

Kennicutt, R. C., Armus, L., Bendo, G. et al. 2003, PASP, 115, 928

Krause, M., Wielebinski, R., \& Dumke, M. 2006, A\&A 448, 133 